\title{
EasiSocial: Recommendations in the Development and Training of Social Media Tools for Older People
}

\author{
Kyle Boyd \\ Ulster University \\ Belfast, BT15 1ED \\ ka.boyd@ulster.ac.uk
}

\author{
Raymond Bond \\ Ulster University \\ Jordanstown,BT37 0QB \\ rb.bond@ulster.ac.uk
}

\author{
Chris Nugent \\ Ulster University \\ Jordanstown,BT37 0QB \\ cd.nugent@ulster.ac.uk
}

\author{
Mark Donnelly \\ Ulster University \\ Jordanstown,BT37 0QB \\ mp.donnelly@ulster.ac.uk
}

\begin{abstract}
Online Social Networks are regarded as one of the biggest successes of the Internet. Facebook, for example, has over 1.59 billion monthly active users. Online Social Networks offer one potential strategy for addressing feelings of social isolation amongst the aging population. Nevertheless, many older people do not use online social networks due to barriers resulting from a lack of understanding, and perceived usefulness of these services. This paper presents the findings from a ten-week training course where participants were trained to use Facebook and introduced to a novel web application, called EasiSocial, which personalises Facebook's user interface (UI), for use by older users. An evaluation study was undertaken with 9 users (69-80 years old, mean age 75.2, ST DEV 3.231) of both services to highlight existing adoption barriers and to validate the hypothesis that personalising the UI of online social media services can promote engagement.. Our analysis demonstrated that EasiSocial was statistically significantly 'easier' ( $p$ value $<0.05$ ) to learn and use than the standard Facebook UI and therefore potentially helpful to increase the adoption of social media technologies. The study also highlighted that training plays an important role in supporting adoption. Recommendations for both development and training are highlighted.
\end{abstract}

Service Design, Facebook, Online Social Networks, Design Recommendations, Social Media, Social isolation, Technology adoption

\section{INTRODUCTION}

Kaplan and Haenlein [1] define social media as a group of Internet technologies built on the platform of Web 2.0 technologies that allows the creation and exchange of user generated content. Web 2.0 is a term that represents those technologies supporting: collaboration, blogs and microblogs, content communities, picture sharing, social networking, virtual gaming and virtual social worlds. Online social networking (OSN) services have emerged as a direct result of the interactivity supported by Web 2.0 and is now regarded as one of the most impactful online services. OSNs allow for people to build social relations who share interests, activities and friendships. Facebook, for example, attracts approximately 1.59 billion users each month [2].

Currently, in the United Kingdom (UK), 11.4 million people are aged 65 and over [3]. The number of people aged 65+ is projected to rise by over 40 per cent $(40.77 \%)$ in the next 17 years to over 16 million [3]. Traditionally, "old age" has been associated with the negative stereotypes of frailty, ill health and social and economic dependency, which can lead to social isolation. Previous research has suggested that Information and Communication Technology (ICT) and the facet of social media, online social networks could be used to help 'connect' those who are socially isolated to enable them to maintain regular contact with family and friends[4]-[6] and indeed evidence suggests that there are any social and cognitive benefits for older adults when they use this technology to create content and participate in information sharing with family and friends [7]-[9]. Social interactions allow people to share knowledge, expertise, new skills and help them feel that they are contributing to society as a whole [9]-[12]. Nevertheless, barriers to technology adoption among older people have been reported. Reasons are attributed to lower levels of education and a lack of formal training provision for older people [13], [14]. In our previous work, we investigated the specific user needs of older people in the training and usage of Facebook [15], [16] by conducting a usability test. Facebook was chosen as the starting point given that it is the biggest OSN and as reported by Smith the mostly likely to be used by older people [2], [17]. The results from this study confirmed that existing OSNs do not needs of older people and led to the establishment of a Usability Protocol [18].

Emerging from our initial research was the design and implementation of EasiSocial, which translated the identified user needs of older users into an approachable and personalisable UI for interfacing with Facebook. EasiSocial aims to maximise user experience by enforcing strong learnability characteristics. The present study presents our methodology and evaluation of EasiSocial over a two and half month period. 


\section{Methods}

This section details how the EasiSocial application was designed and implemented, and presents the protocol employed to facilitate the evaluation of EasiSocial.

\subsection{EasiSocial Design and Evaluation}

The EasiSocial web application was focused on design and usability. Specifically, EasiSocial attempts to tailor the interaction and usability of Facebook for to an older novice user group by providing an UI skin over the current OSN.

The evaluation was conducted with a group of older participants attending a local group SLIG (Suffolk and Lenadoon Interface Group) in Belfast, Northern Ireland. Appropriate ethical approval was sought prior to engaging with user that led to the recruitment of nine participants (mean age 76.5). During the study, the participants were given each application, Facebook and EasiSocial to use at home for two weeks.

\section{RESULTS}

Below are the results from the evaluation. During the evaluation, core metrics were assessed; time spent using technology and subjective satisfaction. At the end of the study, the data collected was investigated to assess whether prior training assisted in the adoption and use of the web application[19].

\subsection{The usability questionnaire results}

During the period when the participants used the tablets at home they were given a usability questionnaire to complete at the end of each week. This was an important part of understanding how usable both applications were. The usability questionnaire had 30 questions in a 7-point Likert scale relating to the usefulness, ease of use, ease of learning and satisfaction of the applications. The findings are presented in Table 3.

The null hypothesis was to see if there was any difference in Facebook and the tailored EasiSocial solution. Even when all the usability metrics are combined (Figure 1) (Usefulness, Ease of Use, Ease of Learning, Satisfaction.) EasiSocial scores higher than Facebook. This would suggest that from a usability standpoint EasiSocial performs better than Facebook.

\subsection{Design and training recommendations}

Two sets of recommendations were created following this study, based on feedback from participants and the observations of the authors. The first is a set of recommendations, presented in Table 5 that outlines UI considerations for OSN development of services for older users. This will help in the design of OSNs and aim to improve their usability amongst older people.

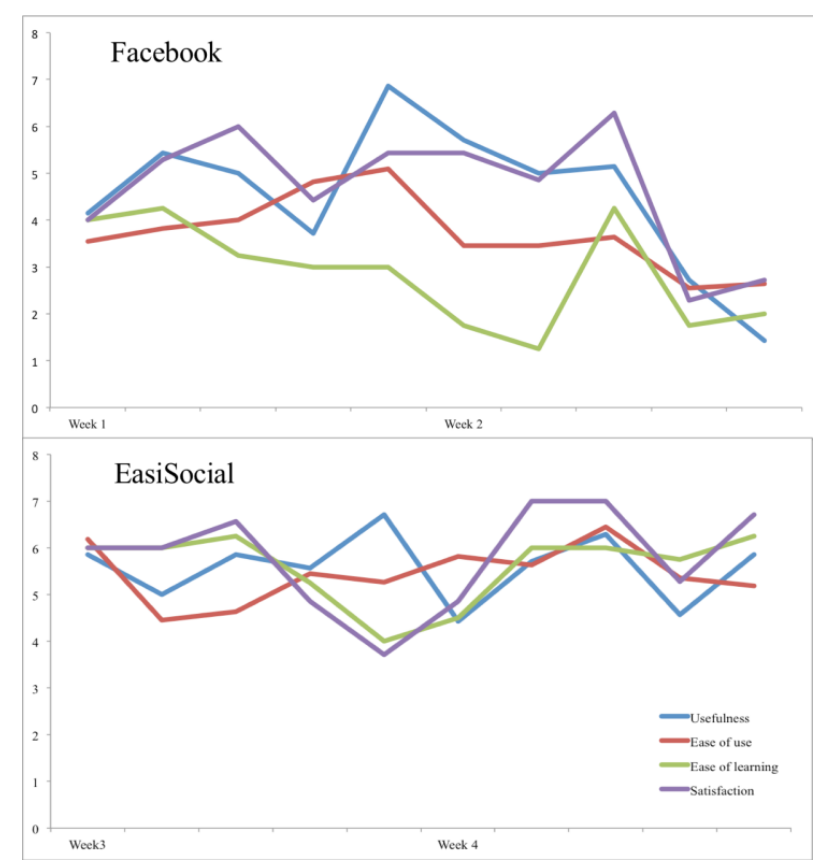

Figure 1 Combining the usability metrics gives a clear indication that overall EasiSocial Scores better then Facebook.

Table 1: User Interface Design recommendations and rationale for designing OSNs for older people.

\section{Layout}

1. Remove all content not necessary to function i.e. Advertisements

Do not include any unnecessary content, which is not needed for the interface to work. Keep it as simple as possible.

\section{Each interface should have one feature}

Each interface should only have one achievable aim. Keep tasks simple, short and narrowly focused.

\section{Minimise visual clutter}

This is a good principle for any interface, however, especially so when considering older participants. Avoid lots of icons throughout the interface.

\section{Use modal context when necessary}

Keep modal tasks simple, short and narrowly focused. Provide a safe way to exit modal.

\section{Functionality}

\section{Have a flat navigation structure with navigation on every page}

In an app with a flat information structure, users can navigate directly from one primary category 
to another given that all primary categories are accessible from the main screen. Users should always know where they are in the application and how to reach their next destination.

\section{No login panel}

Minimise login difficulty through use of a simple login. Logging in automatically would be the preference[15].

\section{Limit features to the most popular}

Limit functionality of the interfaces to the main interactions of the social media technology. Focus on what you are trying to adapt. This helps to maximise understanding and purpose of how it works. Focus on solutions not features.

\section{Limit amount of steps to complete a task}

Try and reduce the number of interactions is takes to complete a task. This makes it easier to remember how to use features.

\section{Go full screen}

Full-Screen windows help users concentrate and make it easy to enter a distraction-free environment that helps focus on a task.

\section{Interactions}

\section{Only make buttons clickable - do not use drag and drop}

If an element is clickable, for example, it must appear that way, or a user may never try clicking it.

11. Make use of the pinch gesture: allowing for quick magnification, however, avoid other gestures

This was valuable for those with visual impairment. It allowed them to quickly view difficult to view parts of the interface.

\section{Provide feedback}

Feedback helps users know what an application is doing. For example if they encounter an error focus on the error, explain what has gone wrong and provide a solution to remedy this[22].

\section{Colour/Language}

13. Use only real world language. Use terminology that you're sure your users understand

Use only real world language, buzzwords don't have meaning in the context of an interface, plus to first time users this can add another level of difficultly[22].

\section{Use colours that work together to provide contrast}

Make sure there's enough contrast between the navigation background and the interface, if the contrast is bad then the buttons will be hard for users to see.

\section{Text should always be legible}

Capitalise labels and text, this helps with readability and use the same anti-aliased, easyto-read fonts that users are accustomed to seeing throughout the interface (Lucida Grande, Helvetica.)

The second set of recommendations, presented in Table 6 , is a set of suggestion for engaging with and training older people to use these OSN technologies.

Table 2: Recommendations for training older people how to use social media.

\section{Sessions}

1. Organise a local training course over a period of 10-12 weeks.

This provides the trainers with enough time to cover the aspects of the social media technology, including hardware. After this length of time participants are happy to do their own thing.

\section{Keep each training session to $\mathbf{4 5}$ minutes to} 1 hour.

Keep the session short and concise, shorter sessions help keep attention spans.

3. During the weeks the participants use the technology at home run a 'call in service' at the training location to answer any queries they may have during the week.

This means that if they have any problems with the technology at home they can call in and have it fixed.

4. Be prepared to repeat weekly sessions around depending on how the group is progressing.

Sometimes it is necessary to repeat weekly sessions to help with remembering.

5. Live demonstrations of the technology as a group are positive. For those struggling one to one time is necessary.

Live demonstrations work best, talking out loud with a running commentary helps to keep everyone involved. For those who are not as keen, one to one help is vital.

\section{Technology}

6. Whatever social media technology is being shown organise a weekly training schedule, focusing on the most common aspects of 


\begin{abstract}
the technology.
Only focus on the main features of any social media. For example, if it is Facebook only cover connecting to friends, updating status and commenting. If it were Instagram, simply show how to connect with friends and upload photos. This limited functionality is enough to cover and it allows participants to gain an understanding of how social media works, whilst also building their network.
\end{abstract}

\section{Only introduce one feature of the social} media technology per week.

One feature per week. Anymore and this can be too detailed for older participants. It becomes confusing and they have forgotten it by the next session.

8. Set up accounts before first training session, minimising risk of dropouts.

Setup the social media accounts for each participant after the initial visit when recruiting participants; this can help reduce the risk of dropout. Registration processes are notoriously difficult. During the first session provide participants with their account details.

9. Tablet touch screen devices with a mobile data connection are recommended. It means participants can use these at home even without a broadband connection.

This is of great benefit. A tablet with its own internet connection removes a lot of barriers that a PC computer would cause for older people. It means they don't have to have an internet connection, however, the tablet is also easy to carry.

10. Use tablet pointers.

The pointer helps to make better interactions. Participants

11. Keep participants around the same age, this limits embarrassment if mistakes are made.

This helps participants not to have anxiety or embarrassment during the training.

\section{For any more than five participants have at} least two educators.

This is necessary to cope with showing the technology, however, also dealing with problems one to one.

13. For those struggling organise a buddy system so participants can help one another.

This is aimed at participants who may have missed a week due to unforeseen circumstances. A more confident participant can help them to catch up.
14. Encourage participants at the training group to connect with one another. This is a good way to build up their social network quickly.

This helps participants to understand how social media works and particularly online social networks if they can see connections being made as they are using the technology.

\section{Remember the social impact.}

These training sessions may be the only social interaction that an older person has from one week to the next. Allow time for general conversation and social time during the training session itself. The well-being of the participants is paramount.

\section{CONCLUSIONS}

The study presented in this paper considered the aims, design, development and evaluation of the EasiSocial web application.

Two sets of recommendations were created following this study. The first is a set of recommendations, presented in Table 1 that outlines UI considerations for OSN development of services for older users. This will help in the design of OSNs and aim to improve there usability amongst older people. The second set of recommendations in Table 2 help with the training needed for older people to help aid adoption[20].

\section{REFERENCES}

[1] A. M. Kaplan and M. Haenlein, "Users of the world, unite! The challenges and opportunities of Social Media," Bus. Horiz., vol. 53, no. 1, pp. 59-68, 2010.

[2] Facebook., "Facebook Home Page." [Online]. Available: http://www.facebook.com Accessed $31 \mathrm{My}$ 2018.

[3] Age UK, "Later Life in the United Kingdom," no. February, pp. 1-25, 2016.

[4] S. Goswami, F. Köbler, J. M. Leimeister, and $\mathrm{H}$. Krcmar, "Using Online Social Networking to Enhance Social Connectedness and Social Support for the Elderly," in Proceedings of the International Conference on Information Systems (ICIS), 2010, vol. 10, pp. 107-132.

[5] R. E. Wilson, S. D. Gosling, and L. T. Graham, "A review of Facebook research in the social sciences," Perspect. Psychol. Sci., vol. 7, no. 3, pp. 203-220, 2012.

[6] A. K. Leist, "Social Media Use of Older Adults: A Mini-Review," Gerontology, vol. 59, no. 4, pp. 378-384, 2013. 
[7] R. Beacker, K. Sellen, S. Crosskey, V.

Boscart, and B. Barbosa Neves,

"Technology to reduce social isolation and Ioneliness," Proc. 16th Int. ACM

SIGACCESS Conf. Comput. Access. -

ASSETS '14, no. November 2015, pp. 2734, 2014.

[8] R. Cornejo, M. Ten Tori, and J. Favela, "Enriching in-person encounters through social media: A study on family

connectedness for the elderly," Int. J. Hum. Comput. Stud., vol. 71, no. 9, pp. 889-899, 2013.

[9] J. W. Myhre, M. R. Mehl, and E. L. Glisky, "Cognitive Benefits of Online Social Networking for Healthy Older Adults," Journals Gerontol. Ser. B Psychol. Sci. Soc. Sci. , Mar. 2016.

[10] B. O'Dea and A. Campbell, "Healthy connections: online social networks and their potential for peer support," Stud. Health Technol. Inform., vol. 168, pp. 133140, 2011.

[11] R. Grieve, M. Indian, K. Witteveen, G. Anne Tolan, and J. Marrington, "Face-to-face or Facebook: Can social connectedness be derived online?," Comput. Human Behav., vol. 29, no. 3, pp. 604-609, May 2013.

[12] D. Richter, S. Bannier, R. Glott, M. Marquard, and T. Schwarze, "Universal Access in Human-Computer Interaction. User and Context Diversity: 7th International Conference, UAHCI 2013, Held as Part of HCI International 2013, Las Vegas, NV, USA, July 21-26, 2013, Proceedings, Part II," C. Stephanidis and M. Antona, Eds. Berlin, Heidelberg: Springer Berlin Heidelberg, 2013, pp. 211-220.

[13] M. Y. Chan, S. Haber, L. M. Drew, and D. C. Park, "Training Older Adults to Use Tablet Computers: Does It Enhance Cognitive Function?," Gerontol. , Jun. 2014.

[14] A. Concern, "Older People and digital inclusion, http://intelli.ageuk.org.uk/e/d.dll?m=1292\&ur I=http://www.ageuk.org.uk/documents/en$\mathrm{gb} /$ for-professionals/computers-andtechnology/older\%20people\%20and\%20digi tal\%20inclusion.pdf?dtrak=true, Accessed 07/04/2011. ,"Age Concern, 2010.
[15] K. Boyd, C. Nugent, M. Donnelly, R. Bond, R. Sterritt, and L. Gibson, "Investigating Methods for Increasing the Adoption of Social Media amongst Carers for the Elderly," in XIII Mediterranean Conference on Medical and Biological Engineering and Computing 2013, 2014, pp. 1439-1442.

[16] C. Bell, C. Fausset, S. Farmer, J. Nguyen, L. Harley, and W. B. Fain, "Examining social media use among older adults," in Proceedings of the 24th ACM Conference on Hypertext and Social Media, 2013, pp. 158-163.

[17] A. Smith, "Older Adults and Technology Use," Pew Res. Cent., no. April, pp. 1-26, 2014.

[18] K. Boyd, C. Nugent, M. Donnelly, R. Sterritt, and R. Bond, "A usability protocol for evaluating online social networks," Impact Anal. Solut. Chronic Dis. Prev. Manag., vol. Lecture No, pp. 222-225, 2012.

[19] T. Tullis and W. Albert, "Measuring the user experience," in Measuring the User Experience: Collecting, Analyzing, and Presenting Usability Metrics, 2008, pp. 142144.

[20] J. Coelho and C. Duarte, "A literature survey on older adults' use of social network services and social applications," Comput. Human Behav., vol. 58, pp. 187-205, May 2016. 